\title{
Waveform design for integration of MIMO radar and communication based on orthogonal frequency division complex modulation
}

\author{
Xiaomeng Cui ${ }^{1,2}$, Jie Yang ${ }^{1, *}$, Tao Yan ${ }^{2}$, and Ruijuan Yang $^{2}$ \\ ${ }^{1}$ Wuhan University of Technology, Wuhan, China \\ ${ }^{2}$ Air Force Early Warning Academy, Wuhan, China
}

\begin{abstract}
The integration technology of radar and communication is an effective way to realize resource platform sharing, system advantages complementation and comprehensive performance improvement. Aiming at the shortcomings of the current integrated waveform, an integrated waveform for MIMO radar and communication is proposed in the paper based on orthogonal frequency division complex modulation (OFDCM), which is of favourable performance on the radar and communication. Theoretical analysis and simulation experiments show that the designed waveform not only acquires excellent communication performance with high transmission rate and large transmission capacity, but also features satisfactory performance for radar targets detection such as wide bandwidth, interference rejection, low interception and high doppler tolerance.
\end{abstract}

\section{Introduction}

The modern information war has given birth to the rapid development of integration technology of radar and communication. By combining targets detection with information communication, the technology has realized data transmission with high efficiency, realtime, large-capacity and long-distance. At the same time, the integration technology also could reduce system complexity, increase the utilization rate of electronic equipment and spectrum resources, and improve the system operational effectiveness [1].

The key technology of radar-communication integration is to design the signal waveform which can realize radar and communication function simultaneously. At present, the typical integrated waveform can be divided into three categories: (1) the two waveforms are produced independently, the method of multiplexing or superposition is adopted while transmitting, and time-sharing receiving or separation algorithm is adopted in the receiver. A time-division multiplexing integrated waveform based on the continuous wave system is designed in reference [2], but this waveform is characterized by low time resource utilization. Integrated waveform based on frequency division multiplexing is proposed in reference [3], in which the frequency bands are alternately used for radar and communication within the working bandwidth. However, it is difficult to consider both

* Corresponding author: 466802324@qq.com 
communication speed and range resolution, and more the non-continuous band could lead to false peaks after pulse compression. (2) Communication information is modulated on radar waveform. In reference [4], communication information is mapped to LFM signal sequence with different initial frequency, and then integration waveform whose initial frequency is modulated by the communication information is proposed, but it is difficult to balance data transmission rate and signal correlation requirements. In reference [5], the communication information is modulated on the phase of the LFM signal by minimum shift keying (MSK), only one communication message can be transmitted at one time, both the data transmission rate and frequency band utilization rate are very low. (3) Reconstruction of communication waveforms based on radar functions [6,7]. Such waveforms mostly use OFDM signal as the integrated waveform to explore the performance of radar targets detection, which is of excellent communication performance. but it is of poor targets detection performance on distance resolution and doppler sensitivity. In summary, the first type of waveform is only an implementation of radar-communication integration, it not really reflects the essence of "integration". and the latter two types of integrated waveform could not meet the performance demands of both radar and communication. Therefore, one of the difficult points is to seek integrated waveform with both excellent performance of radar and communication.

In this background, an integrated waveform for MIMO radar and communication based on orthogonal frequency division complex modulation (OFDCM) is proposed in the paper. By combining the MIMO-OFDM technology with the signal complex modulation technology, a single transmit signal is modulated with liner frequency on the base of MIMO-OFDM firstly, and then the communication information is modulated to each transmission channel after serial-parallel conversion. The designed integrated signal not only can effectively utilize the spectrum resources and has the advantages of high transmission rate and large transmission capacity, but also could overcome the problem that poor performance of the pure communication signal in radar targets detection.

\section{Signal model and parameters selection}

\subsection{Signal model}

Without loss of generality, it is assumed that $M$ denotes the number of transmission channels (or elements), and $\boldsymbol{s}_{m}(t)(m=1,2, \ldots, M)$ denotes the integrated signal of $m$ th transmission channel, whose time domain model can be expressed as

$$
\boldsymbol{s}_{m}^{\text {OFDCM }}(t)=a_{m}(t) \exp \left[j 2 \pi\left(f_{0}+k_{m} \Delta f\right) t\right] \exp \left[j \varphi_{m}(t)\right] \exp \left(j \pi \mu t^{2}\right) \quad 0<t<T_{p}
$$

where $a_{m}(t)$ denotes the amplitude modulation function of $m$ th integrated signal, here rectangular pulse function is selected, that is $a_{m}(t)=\left(T_{p}\right)^{-1 / 2} \operatorname{rect}\left(t / T_{p}\right)$. The $f_{0}+k_{m} \Delta f$ denotes the frequency of $m$ th integrated signal, in which $f_{0}$ and $k_{m}$ respectively denote fundamental frequency and the offset of $m$ th transmitting frequency relative to $f_{0}$, here $k_{m}=m-1$. The $\mu=B_{0} / T_{p}$ denotes the frequency modulation rate, where $B_{0}$ and $T_{p}$ respectively denote modulation bandwidth and pulse width of single channel signal. The $\varphi_{m}(t)$ denotes the communication information modulation function of $m$ th integrated signal, Quadrature Phase Shift Keying(QPSK) is adapted here, then equation (1) can be rewritten as

$$
\boldsymbol{s}_{m}^{\text {OFDCM }}(t)=\frac{1}{\sqrt{Q}} \sum_{q=1}^{Q} c_{m, q} v\left(t-q \tau_{c}\right) \cdot \exp \left(j \pi \mu t^{2}\right) \exp \left[j 2 \pi\left(f_{0}+k_{m} \Delta f\right) t\right] \quad 0<t<T_{p}
$$

where $\tau_{c}$ and $Q$ respectively denote sub-pulse width and the length of codes, and here $T_{p}=Q \tau_{c}$. The $c_{m, q}$ denotes the $m$ th communication information code of $m$ th integrated signal, and $c_{m, q}=\exp \left[j \varphi_{m}(q)\right]$. The $v(t)=\left(\tau_{c}\right)^{-1 / 2} \operatorname{rect}\left(t / \tau_{c}\right)$ denotes amplitude function of sub-pulse. 


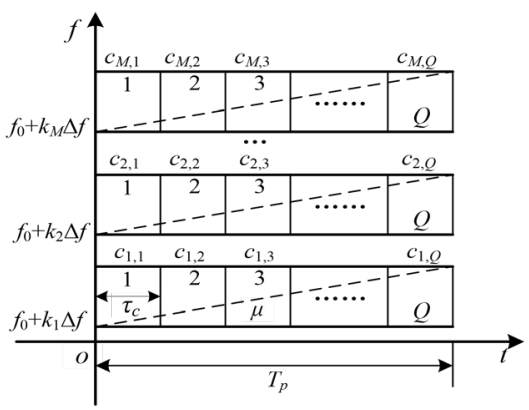

Fig. 1. Structure principle of OFDCM integrated waveform.

The structure principle of OFDCM integrated waveform is shown in figure 1, it can be seen that OFDCM waveform can be considered as communication information modulation on the basis of OFDM-LFM waveform for MIMO radar, and it can also be considered that the linear frequency modulation based on OFDM-QPSK communication signals.

\subsection{Parameter selection illustration}

\subsubsection{Frequency, time width and bandwidth}

Although there are different working frequency bands for radar and communication, there are overlapping parts in some frequency bands. So, the frequency of integrated waveform should be chosen in the public spectrum of radar and communication. In order to ensure high range resolution and high communication speed, big bandwidth with hardware implementation is selected as far as possible. Similarly, the time width selection should be based on radar detection range to ensure radar range.

\subsubsection{Number of transmitting channels and frequency interval}

In general, the more the number of transmitting channels, the higher the communication transmission rate, and the lower the receiver dynamic range requirements, but it also increases the system complexity. In order to maintain the orthogonality between the transmit channels, here the frequency band interval of each channel is selected as $\Delta f=B_{0}$, that is to see the frequency interval is equal to the bandwidth of single channel.

\subsubsection{Code generation}

The four-phase encoding is selected as the basic information code element. In MIMO radar, a great deal of references has been devoted to the design of quadrature phase coding It is usually realized through optimization algorithm, which can achieve good correlation characteristics at the expense of large computation and poor real-time performance. The chaotic signals [8] are widely used in radar waveform design due to the excellent correlation characteristics and real-time performance, in this background, a new hybrid chaotic map based on Chebyshev mapping and Tent mapping is constructed in this paper, whose iterative model can be expressed as

$$
x_{n+1}=f\left(x_{n}\right)=\left\{\begin{array}{c}
\cos \left(\mu_{1} \cdot \arccos \left(x_{n}\right)\right),-1<x_{n} \leq 0 \\
1-\mu_{2}\left|x_{n}\right|, 0<x_{n}<1
\end{array}\right.
$$

where $\mu_{1}$ and $\mu_{2}$ denote mapping parameter, here $\mu_{1}=2.5$ and $\mu_{2}=1.999$. A set of random signals can be generated by choosing different $x_{0}$ and number of iterations. 


\section{Performance analysis}

The performance of the designed OFDCM integrated waveform will be analyzed from radar and communication in the section.

\subsection{Radar performance analysis}

Ambiguity function of MIMO radar can be expressed as [9]

$$
\chi_{m, m^{\prime}}(\Delta \tau, \Delta v)=\int_{-\infty}^{\infty} u_{m}(t) u_{m^{\prime}}^{*}(t+\Delta \tau) \exp (j 2 \pi \Delta v t) d t
$$

Substituting equation (2) into equation (4) can achieve follow equation

$$
\chi_{m, m^{\prime}}^{O F D M}(\Delta \tau, \Delta v)=\chi_{m, m^{\prime}}^{P C}\left(\Delta \tau, \Delta v-\mu \Delta \tau+\left(k_{m}-k_{m^{\prime}}\right) \Delta f\right) \exp \left[-j \pi \mu(\Delta \tau)^{2}\right] \exp \left[-j 2 \pi k_{m^{\prime}} \Delta f \Delta \tau\right]
$$

Let $m=m^{\prime}$ and $\Delta v=0$ in equation (5), the range autocorrelation function can be shown as

$$
\left|\chi_{m}^{O F D C M}(\Delta \tau, 0)\right|=\left|\chi_{m}^{P C}(\Delta \tau,-\mu \Delta \tau)\right|
$$

Then the range resolution can easily be deduced as

$$
\rho=\frac{c}{2} \cdot \frac{A_{\Delta \tau}}{2}=\frac{c \tau_{c}}{2\left(B_{0}^{2} \tau_{c}^{2}+1\right)^{1 / 2}}
$$

From equation (7) we can see that the range resolution of integrated waveform is approximately equal to LFM waveform $\left(c / 2 B_{0}\right)$, and it is less than range resolution of phase code $\left(c \tau_{c} / 2\right)$. Let $m=m^{\prime}$ and $\Delta \tau=0$ in equation (5), then the velocity autocorrelation function can be shown as

$$
\left|\chi_{m}^{O F D C M}(0, \Delta v)\right|=\left|\chi_{m}^{P C}(0, \Delta v)\right|
$$

It is observed that the velocity resolution of integrated waveform is equal to phase code waveform $\left(1 / Q \tau_{c}\right.$ or $\left.1 / T_{p}\right)$, which is only relative to pulse width.

\subsection{Communication performance analysis}

\subsubsection{Data rate}

Data rate(DR) represents the amount of data transmitted in unit of time (per second). The data transmission rate of the designed integrated signal in the paper can be expressed as

$$
R_{b}=\frac{M}{\tau} \log _{2}^{N}
$$

where $N$ denotes communication encoding mechanism, here $N=4$. It is observed that the data transmission rate of designed integrated signal is $M$ times more than single frequency serial transmission.

\subsubsection{Symbol error rate}

The symbol error rate(SER) is an indicator to measure the accuracy of communication transmission in specified time. In the paper, symbol error rate of coherent demodulation under gaussian white noise channel can be expressed as

$$
P_{e}=1-\left\{1-\frac{1}{2} \operatorname{erfc}\left[\left(\mathrm{SNR}_{\mathrm{I}} / 2\right)^{1 / 2}\right]\right\}^{2}
$$

Here $\mathrm{SNR}_{\mathrm{I}}$ denotes input SNR. 


\section{Simulation experiment and analysis}

In this section, computer simulations for the proposed OFDCM integrated waveform are conducted, the corresponding parameters set as follows.

Table 1. Simulation parameters.

\begin{tabular}{|l|l|l|l|l|}
\hline$M$ & $f_{0}$ & $t_{p}$ & $B_{0}$ & $Q$ \\
\hline 4 & $2 \mathrm{GHz}$ & $80 \mu \mathrm{s}$ & $20 \mathrm{MHz}$ & 128 \\
\hline
\end{tabular}

\subsection{Integrated waveform simulation}

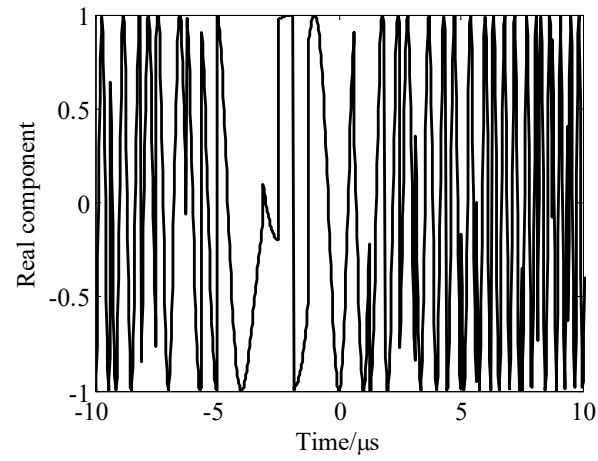

(a)Real component

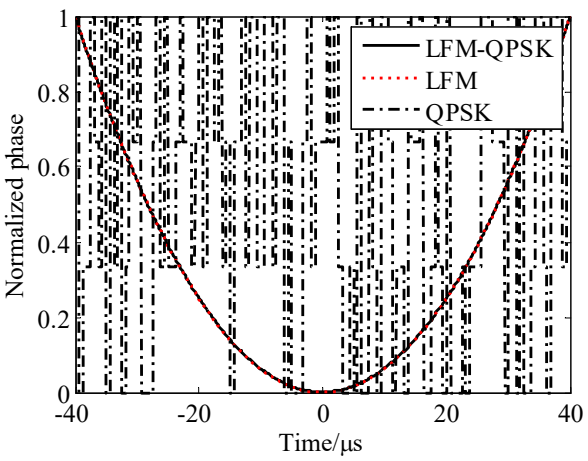

(b)Phase

Fig. 2. Integrated waveform simulation of single channel.

Figure 2 shows time domain simulation of the designed integrated waveform (here the first transmission channel is taken as an example), in which figure 2 (a) and figure 2 (b) respectively represents the real component and phase. It can be seen that the real part and phase of integrated waveform keep the shape and changing tendency of the LFM signal as a whole, but the mutation occurs due to the modulation of communication code, which changing the continuously changing characteristics of real part and phase for LFM waveform.

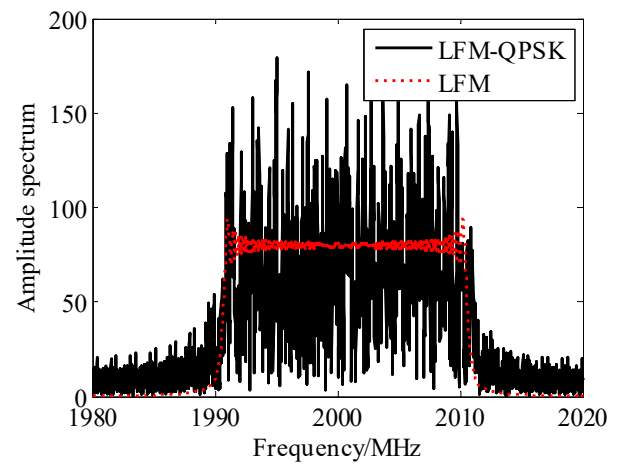

(a) Single channel spectrum

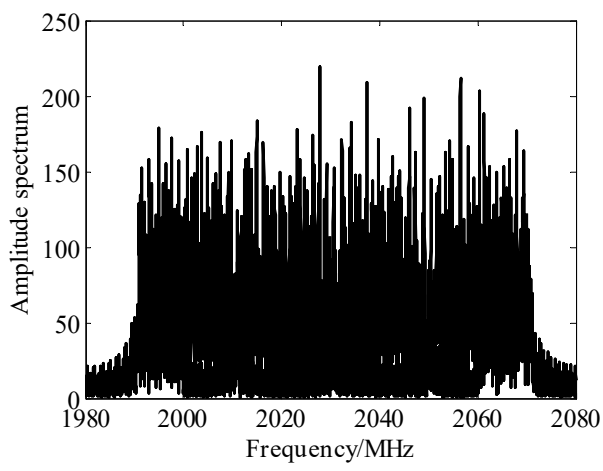

(b)Entire signal spectrum

Fig. 3. Integrated waveform spectrum.

The spectrum of integrated waveform is shown in figure 3, in which figure 3 (a) shows the single channel spectrum. It can be seen that the spectrum appears as a rectangular envelope in the frequency range and the envelope width is approximately equal to the frequency bandwidth. This is also similar to the LFM signal spectrum, but the in-band spectrum is irregularly distributed due to the modulation of the communication code. The 
entire spectrum of multi-channel integrated signal is shown in figure 3 (b), because the frequency interval of different transmission channels is exactly equal to the single channel FM bandwidth, so the entire bandwidth of integrated waveform is $80 \mathrm{MHz}$.

\subsection{Comprehensive performance simulation}

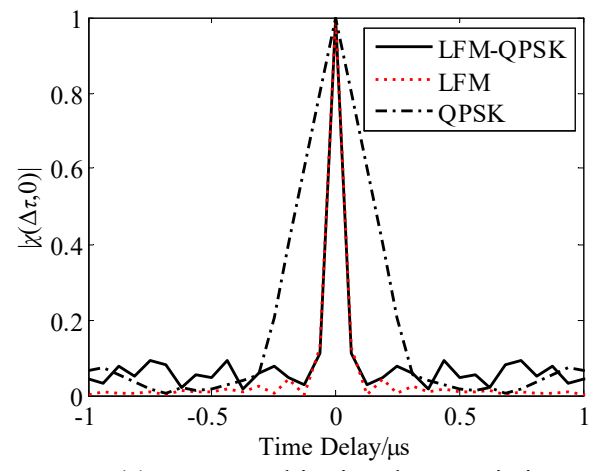

(a) Range ambiguity characteristics

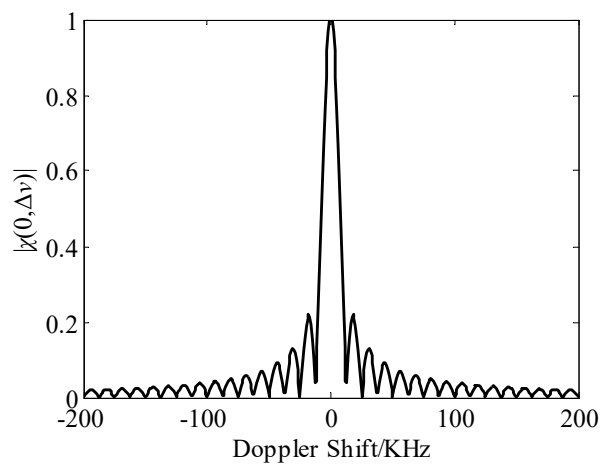

(b) Velocity ambiguity characteristics

Fig. 4. Ambiguity characteristics of OFDCM integrated waveform.

The ambiguity function simulation of single channel integrated waveform is given in figure 4 , in which figure 4(a) and figure 4(b) denote the range ambiguity function and velocity ambiguity function respectively. It can be seen that main lobe width of distance ambiguity function for integrated signal is similar with LFM waveform, but far less than the pure communication coded signal. The simulation results shown in figure 4 verify the theoretical analysis in section 3.1 .

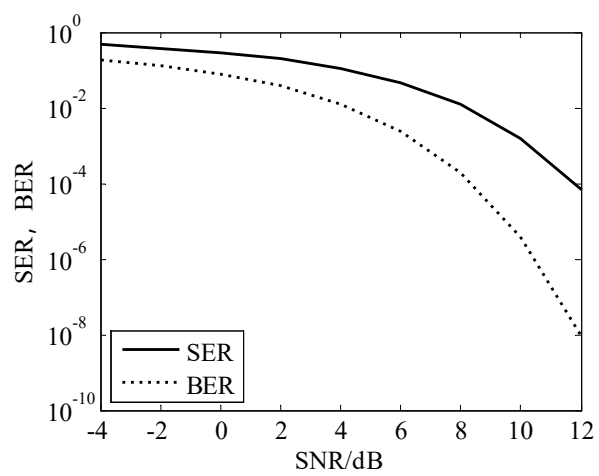

Fig. 5. SER and BER of OFDCM integrated waveform.

The SER and BER (bit error rate) of OFDCM integrated waveform is shown in figure 5, It can be seen that OFDCM integrated waveform acquires low SER and BER. The SER and BER were reduced with the increase of SNR. Combining the above simulation parameters and assuming a duty cycle of 0.3 for the waveform, then the transmission rate of the integrated waveform can be calculated to be $3.84 \mathrm{Mbs}^{-1}$, while the transmission rate with the same parameters (reference [5]) is only $0.96 \mathrm{Mbs}^{-1}$. In fact, in order to simplify the explanation, only four transmitting channels are selected in this paper, but more channels are often used in practice, which can increase the signal transmission efficiency exponentially.

\section{Conclusion}


The integration of radar and communication is an important direction in the development of radar and communication technology in recent years, and it is also an inevitable trend of multi-functional integrated electronic system. Aiming at the shortcomings of existing radar integrated waveforms, an integrated waveform based on OFDCM is proposed in the paper. Firstly, the mathematical model of OFDCM integrated waveform is established. And then the waveform performance of radar and communication are analysed from the aspects of ambiguity characteristic, data transmission rate and bit error rate, after that the simulation analysis are carried out. Theoretical analysis and simulation experiments verify the excellent performance in both of radar and communication.

\section{Acknowledgments}

This work is supported by the National Natural Science Foundation of China (grant 61271451).

\section{References}

1. X. B. Chen, X.M. Wang, C. Cao, et al. Techniques analysis of radar-communication integrating waveform[J]. Modern Radar, 35 (12):56-59. (2013)

2. L. Han and K. Wu. $24 \mathrm{GHz}$ integrated radio and system capable of time agile wireless communication and sensing[J]. IEEE Trans. On Microwave Theory and Techniques, 60 (3):619-631. (2012)

3. X.B. Li, R.J. Yang, W. Cheng. Integrated radar and communication based on multicarrier frequency modulation chirp signal[J]. Journal of Electronics \& Information Technology, 35 (2):406-412. (2013)

4. X.B. Li, R.J. Yang, W. Cheng. The sharing signal for integrated radar and communication based on FRFT[J]. Signal Processing, 28 (4):24-28. (2012)

5. Z.P. Liu, X.B. Chen, X.M. Wang, et al. Communication analysis of integrated waveform based on LFM and MSK[C].2015 IET Radar Conference, Hangzhou, China:1-5. (2015)

6. Y.L. Sit and T. Zwick. MIMO OFDM radar with communication and interference cancellation features[C]. 2014 IEEE Radar Conference, Cincinnati, USA:19-23. (2014)

7. Y.J. Liu, G.S. Liao, Z.W. Yang, et al. A super-resolution design method for integration of OFDM radar and communication[J]. Journal of Electronics and Information Technology, 38 (2):425-433. (2016)

8. B. Chen. Assessment and improvement of autocorrelation performance of chaotic sequences using a phase space method[J]. Science China Information Sciences, 54 (12): 2647-2659. (2011)

9. C.Y. Chen, P.P. Vaidyanathan. MIMO radar ambiguity properties and optimization using frequency-hopping waveforms[J]. IEEE Transactions on Signal Processing, 56 (12):5926-5936. (2008) 